TRANSACTIONS OF THE

AMERICAN MATHEMATICAL SOCIETY

Volume 361, Number 3, March 2009, Pages 1317-1332

S 0002-9947(08)04685-0

Article electronically published on October 16, 2008

\title{
FINITENESS OF STATIONARY CONFIGURATIONS OF THE FOUR-VORTEX PROBLEM
}

\author{
MARSHALL HAMPTON AND RICHARD MOECKEL
}

\begin{abstract}
We show that the number of relative equilibria, equilibria, and rigidly translating configurations in the problem of four point vortices is finite. The proof is based on symbolic and exact integer computations which are carried out by computer. We also provide upper bounds for these classes of stationary configurations.
\end{abstract}

\section{INTRODUCTION}

This paper began its life as a companion paper to [13, in which we prove similar results for relative equilibria in the Newtonian four-body problem. The details of the methods which are common to both problems are described in 13. There are interesting differences as well in both the results and techniques used between the vortex problem and the Newtonian case, which we will explain.

A system of $n$ planar point vortices with vortex strengths $\Gamma_{i} \neq 0$ and positions $x_{i} \in \mathbb{R}^{2}$ evolves according to

$$
\Gamma_{j} \dot{x}_{j}=J \nabla_{j} H, \quad 1 \leq j \leq n,
$$

where $J=\left(\begin{array}{cc}0 & 1 \\ -1 & 0\end{array}\right), H=\sum_{i<j} \Gamma_{i} \Gamma_{j} \log r_{i j}$, and $\nabla_{j}$ denotes the two-dimensional partial gradient vector with respect to $x_{j}$. This system was introduced by Helmholtz [15. to model a two-dimensional slice of columnar vortex filaments, with some refinements by Lord Kelvin [32] and Kirchhoff [18]. In general the $n$-vortex system is simpler than the $n$-body problem of point masses governed by Newtonian gravity for a given $n$. For example the three-vortex system is always integrable, whereas in the Newtonian three-body problem there are chaotic regimes 22. Somewhat offsetting this relative simplicity is the larger set of parameter values that we must investigate (since $\Gamma_{i}<0$ is allowed).

Received by the editors December 15, 2006.

2000 Mathematics Subject Classification. Primary 70F10, 70F15, 37N05, 76Bxx.

Key words and phrases. Relative equilibria, vortices.

The first author was partially supported by NSF grant DMS-0202268. The second author was partially supported by NSF grant DMS-0500443.

(C)2008 American Mathematical Society Reverts to public domain 28 years from publication 
It is sometimes more convenient to consider the vortex positions as complex numbers $z_{i}$, in which case the dynamics are given by $\dot{z}_{i}=V_{i}$ where

$$
V_{i}=I \sum_{j \neq i} \frac{\Gamma_{j}}{\overline{z_{i}}-\overline{z_{j}}}
$$

where the overbar denotes complex conjugation and $I^{2}=-1$.

Following O'Neil [26] we will call a configuration stationary if the relative shape remains constant, i.e., if the ratios of intervortex distances remain constant (such solutions are often called homographic). In [26] it is shown that the only stationary configurations of vortices are relative equilibria (uniformly rotating configurations), equilibria, rigidly translating configurations, and collapse configurations. We will not discuss collapse configurations here.

Equilibria are solutions of the equations $V_{i}\left(z_{1}, \ldots, z_{n}\right)=0, i=1, \ldots, n$. Rigid translations satisfy $V_{i}\left(z_{1}, \ldots, z_{n}\right)=V$, where $V \neq 0$ is a common velocity. Both of these equations involve only the conjugate variables, $\overline{z_{i}}$. Alternatively, we can take the conjugate equations which are rational functions of $z_{i}$. After clearing denominators, these equations can be handled directly with the methods of complex algebraic geometry, as in 26. We are able to use symbolic computation to settle a few questions not handled by O'Neil in the case $n=4$.

Relative equilibrium solutions are more complicated. If we set $z_{i}(t)-c=$ $e^{I \lambda t}\left(z_{i}(0)-c\right)$, where $c \in \mathbf{C}$ is a center of rotation and $\lambda \in \mathbf{R}$ is a constant angular velocity, we find that the initial positions must satisfy

$$
\lambda\left(z_{i}-c\right)=-I V_{i}=\sum_{i \neq j} \frac{\Gamma_{i}\left(z_{i}-z_{j}\right)}{r_{i j}^{2}}, \quad 1 \leq j \leq n,
$$

where $r_{i j}^{2}=\left|z_{i}-z_{j}\right|^{2}=\left(z_{i}-z_{j}\right)\left(\overline{z_{i}}-\overline{z_{j}}\right)$. These equations can also be viewed as describing central configurations of point masses attracted by a logarithmic potential. These equations involve both $z_{i}$ and $\bar{z}_{i}$ and so do not admit such a direct attack. However, as noted by O'Neil, the collinear case can be formulated in terms of real polynomial equations.

The three-vortex case was extensively studied by Gröbli [12], although much of that work was unwittingly repeated by Synge 31] and Novikov 25]. Equilateral triangles are always relative equilibria. There are also collinear equilibria and/or relative equilibria, which are determined by a cubic equation in a shape parameter with coefficients which are linear in the vortex strengths. There can be one to three collinear relative equilibria. These results are nicely summarized in $[5]$.

It turns out that $\Gamma=\sum_{i=1}^{n} \Gamma_{i}=0$ is a necessary condition on the vorticities for the existence of rigidly translating solutions. O'Neil 26] proved that for almost every such choice of vortex strengths, there are exactly $(n-1)$ ! rigidly translating configurations. He shows that for almost every choice of vortex strengths satisfying the necessary conditions $L=\sum_{i<j} \Gamma_{i} \Gamma_{j}=0$, there are $(n-2)$ ! equilibria. For the four-vortex case, the equilibria can be found explicitly (cf. Section 2). O'Neil also provides an upper bound of $n ! / 2$ collinear relative equilibria for almost every choice of vortex strengths. He gives simple conditions for finiteness of the number of collinear relative equilibria: it suffices to assume that for $i, j$, and $k$ distinct elements of $\{1,2,3,4\}$ that $\Gamma_{i} \neq 0, \Gamma \neq 0, L \neq 0, \Gamma_{i}+\Gamma_{j}+\Gamma_{k} \neq 0, \Gamma_{i}+\Gamma_{j} \neq 0$, and $\Gamma_{i} \Gamma_{j}+\Gamma_{i} \Gamma_{k}+\Gamma_{j} \Gamma_{k} \neq 0$. 
Relative equilibria of the four-vortex problem are solutions of the algebraic equations (3). It is easy to see that the solution set is invariant under rotations, translations and dilations in the plane. Call two configurations of four points in $\mathbf{R}^{2}$ equivalent if they are related by these symmetry operations. However, we do not view reflected configurations as equivalent. A configuration will be called strictly planar if it is planar but not collinear. Then our main result is:

Theorem 1. If the vorticities $\Gamma_{i}$ are nonzero, then the four-vortex problem has:

(i) exactly 2 equilibria when the necessary condition $L=0$ holds,

(ii) at most 6 rigidly translating configurations when the necessary condition $\Gamma=0$ holds,

(iii) at most 12 collinear relative equilibria,

(iv) at most 14 strictly planar relative equilibria when $\Gamma=0$,

(v) at most 74 strictly planar relative equilibria when $\Gamma \neq 0$ provided $\Gamma_{i}+\Gamma_{j} \neq$ 0 and $\Gamma_{i}+\Gamma_{j}+\Gamma_{k} \neq 0$ for all distinct indices $i, j, k \in\{1,2,3,4\}$.

We are currently unable to handle the relative equilibrium problem when $\Gamma \neq 0$ but one or more of the mass sums $\Gamma_{i}+\Gamma_{j}, \Gamma_{i}+\Gamma_{j}+\Gamma_{k}$ vanishes. Thus, it is possible that such vorticities lead to a continuum of equivalence classes of relative equilibria. This seems unlikely but it is worth noting that in the case of five vortices this phenomenon does indeed occur for certain choices of the vorticities. Namely, four vortices of strength $\Gamma_{1}$ arranged in a rhombus with a fifth vortex of strength $-\Gamma_{1} / 2$ at the center always form a relative equilibrium, no matter the shape of the rhombus [28]. Our methods break down on similar cases in the Newtonian problem (in which a sum of a subset of parameters vanishes). However in the Newtonian problem we are usually only interested in positive parameters (the masses), and so we ignored such cases. Recently, O'Neil has developed a different proof of finiteness for relative equilibria which works for generic vorticities [27]. His genericity assumptions include the nonvanishing mass sum conditions we use.

The technique of proof will vary for the different cases. For strictly planar relative equilibria with a total vorticity $\Gamma \neq 0$ we will closely follow the ideas of [13, which are described in much more detail there. These ideas can also be applied to some of our remaining cases but the alternatives seem simpler. For strictly planar relative equilibria with $\Gamma=0$ and for collinear relative equilibria, a direct attack with resultants succeeds after an appropriate choice of variables and equations. The collinear problem and the cases of equilibria and rigidly translating configurations were already studied by O'Neil [26] for $n$ generic vorticities. We use a different approach for $n=4$ and only need to assume $\Gamma_{i} \neq 0$.

The case of collapse configurations will not be studied in this paper as it possesses a different character than the other types of stationary configurations. There are no collapse configurations unless $L=0$, but if that condition is satisfied it appears that there is usually a one-dimensional set of equivalence classes of collapse configurations [26].

The proofs below are based on symbolic computations. Details of these can be found in an accompanying Mathematica notebook [14.

\section{EQUilibria}

It is considerably easier to analyze the equilibria of vortices compared to relative equilibria. O'Neil argues that there are generically $(n-2)$ ! equilibria for the $n$-vortex 
problem. For four vortices, he derived explicit formulae for the two equilibria [26]. We will derive similar formulas and also verify that the resulting equilibrium points are always finite, noncollision configurations.

To derive these results note that the equilibria are the common zeros of velocities or equivalently of their complex conjugates

$$
\bar{V}_{i}=-I \sum_{j \neq i} \frac{\Gamma_{j}}{z_{i}-z_{j}}, \quad i=1, \ldots, n .
$$

Here we are assuming that $\Gamma_{i} \in \mathbf{R}$. Dropping the factor of $-I$ and clearing denominators gives $n$ polynomial equations $F_{i}\left(z_{1}, \ldots, z_{n}\right)=0$ where

$$
F_{i}=\sum_{j \neq i} \Gamma_{j} \prod_{k \neq i, j}\left(z_{i}-z_{k}\right), \quad i=1, \ldots, n .
$$

These are homogeneous polynomials of degree $n-2$ in the complex variables $z_{i}$. The complex conjugate variables do not appear.

There are two simple relations among these equations. First

$$
\sum_{i=1}^{n} \Gamma_{i} \bar{V}_{i}=-I \sum_{i, j=1}^{n} \frac{\Gamma_{i} \Gamma_{j}}{z_{i}-z_{j}}=0
$$

since the terms cancel in pairs. Also

$$
\sum_{i=1}^{n} \Gamma_{i} z_{i} \bar{V}_{i}=-I \sum_{i, j=1}^{n} \frac{\Gamma_{i} \Gamma_{j} z_{i}}{z_{i}-z_{j}}=-I \sum_{i<j} \frac{\Gamma_{i} \Gamma_{j}\left(z_{i}-z_{j}\right)}{z_{i}-z_{j}}=-I L,
$$

where

$$
L=\sum_{i<j} \Gamma_{i} \Gamma_{j}
$$

Thus $L=0$ is a necessary condition on the vorticities in order that they admit equilibria.

Assuming $L=0$ we can use any $n-2$ of the equations to determine the equilibria. We are only interested in the solutions of this system with $z \in \mathbf{C}^{n} \backslash \Delta$ where $z=$ $\left(z_{1}, \ldots, z_{n}\right)$ and $\Delta$ is the generalized diagonal: $\Delta=\left\{z: z_{i}=z_{j}\right.$ for some $\left.i \neq j\right\}$. Using translation symmetry we may set $z_{n}=0$. Homogeneity implies that we may view $\zeta=\left(z_{1}, \ldots, z_{n-1}\right)$ as homogeneous coordinates of a point $[\zeta] \in \mathbf{C P}^{n-2}$. If $z \notin \Delta$, then $z_{i} \neq 0, i=1, \ldots, n-1$. Then we can always find a representative for [ל] with $z_{n-1}=1$.

For $n=4$ we are reduced to $n-2=2$ equations for $[\zeta] \in \mathbf{C P}^{2}$ and we may assume $\zeta=\left(z_{1}, z_{2}, 1\right)$. For example, we could use the equations

$$
\begin{aligned}
& F_{3}\left(z_{1}, z_{2}, 1,0\right)=\Gamma_{4} z_{1} z_{2}-\left(\Gamma_{2}+\Gamma_{4}\right) z_{1}-\left(\Gamma_{1}+\Gamma_{4}\right) z_{2}+\left(\Gamma_{1}+\Gamma_{2}+\Gamma_{4}\right), \\
& F_{4}\left(z_{1}, z_{2}, 1,0\right)=\Gamma_{3} z_{1} z_{2}+\Gamma_{2} z_{1}+\Gamma_{1} z_{2} .
\end{aligned}
$$

The resultant of these two equations with respect to $z_{2}$ is

$$
r\left(z_{1}\right)=-\Gamma_{1}\left(\Gamma_{2}+\Gamma_{3}+\Gamma_{4}\right) z_{1}^{2}+\Gamma_{1}\left(2 \Gamma_{4}+\Gamma_{2}\right) z_{1}-\Gamma_{1}\left(\Gamma_{1}+\Gamma_{2}+\Gamma_{4}\right),
$$

where the relation $L=0$ has been used to simplify the coefficients. The roots of this quadratic equation are easily found and then $z_{2}$ is uniquely determined. The equilibria are:

$$
z_{1}=\frac{2 \Gamma_{4}+\Gamma_{2} \pm i \sqrt{3} \Gamma_{2}}{2\left(\Gamma_{2}+\Gamma_{3}+\Gamma_{4}\right)}, \quad z_{2}=\frac{2 \Gamma_{4}+\Gamma_{1} \mp i \sqrt{3} \Gamma_{1}}{2\left(\Gamma_{1}+\Gamma_{3}+\Gamma_{4}\right)} .
$$


Note that these are always distinct for nonzero vorticities. We will now verify that they are finite and do not lie in the diagonal. Both of these questions can be reduced to a simple lemma.

Lemma 1. Let $\Gamma_{1}, \ldots, \Gamma_{4}$ be nonzero and satisfy $L=0$. Then $\Gamma_{1}+\Gamma_{2}+\Gamma_{3}=0$ if and only if $\Gamma_{1} \Gamma_{2}+\Gamma_{1} \Gamma_{3}+\Gamma_{2} \Gamma_{3}=0$ and, in this case, $\Gamma_{i}$ cannot all be real.

Proof. We have $L=\Gamma_{1} \Gamma_{2}+\Gamma_{1} \Gamma_{3}+\Gamma_{2} \Gamma_{3}+\left(\Gamma_{1}+\Gamma_{2}+\Gamma_{3}\right) \Gamma_{4}=0$ and since $\Gamma_{4} \neq 0$, the first statement holds. We will show that $\Gamma_{1}, \Gamma_{2}, \Gamma_{3}$ are the cube roots of some complex number and so are not all real. Indeed, the vanishing quantities are just two of the elementary symmetric functions of $\Gamma_{1}, \Gamma_{2}, \Gamma_{3}$ so

$$
\left(z-\Gamma_{1}\right)\left(z-\Gamma_{2}\right)\left(z-\Gamma_{3}\right)=z^{3}-\Gamma_{1} \Gamma_{2} \Gamma_{3} .
$$

Of course a similar result holds when 1,2,3 are replaced by any three of the four subscripts.

It follows that for real, nonzero vorticities, the denominators in the expressions for $z_{1}, z_{2}$ cannot vanish. Also, we cannot have $z_{i}=0,1$. To see this note that

$$
r(0)=\Gamma_{1}\left(\Gamma_{1} \Gamma_{3}+\Gamma_{1} \Gamma_{4}+\Gamma_{3} \Gamma_{4}\right), \quad r(1)=\Gamma_{2}\left(\Gamma_{2} \Gamma_{3}+\Gamma_{2} \Gamma_{4}+\Gamma_{3} \Gamma_{4}\right) .
$$

Since these do not vanish, $z_{1} \neq 0,1$. A similar argument applied to the resultant $r\left(z_{2}\right)$ with respect to $z_{1}$ shows $z_{2} \neq 0,1$. Thus there are always exactly two equilibria and Theorem 1 (i) is proved.

\section{Rigidly translating SOlutions}

For rigidly translating solutions, all velocities must be nonzero and equal, so there is some complex number $V \neq 0$ such that $\bar{V}_{i}=\bar{V}, i=1, \ldots, n$. From (4) we have

$$
\bar{V} \sum_{i=1}^{n} \Gamma_{i}=0
$$

Thus $\Gamma=\sum_{i=1}^{n} \Gamma_{i}=0$ is a necessary condition for rigid translations to be possible. Relation (5) gives

$$
\bar{V} \sum_{i=1}^{n} \Gamma_{i} z_{i}=-I L .
$$

We can eliminate $V$ by taking differences to get $n-1$ equations

$$
\bar{V}_{i}-\bar{V}_{n}=0, \quad i=1, \ldots
$$

Clearing denominators gives equations $G_{i}\left(z_{1}, \ldots, z_{n}\right)=0, i=1, \ldots, n-1$, where

(6) $G_{i}=\sum_{j \neq i} \Gamma_{j} \prod_{k \neq i, j}\left(z_{i}-z_{k}\right) \prod_{k \neq i, n}\left(z_{n}-z_{k}\right)+\sum_{j \neq n} \Gamma_{j} \prod_{k \neq i, n}\left(z_{i}-z_{k}\right) \prod_{k \neq j, n}\left(z_{n}-z_{k}\right)$.

These are homogeneous polynomials of degree $2 n-4$. If we assume $\Gamma=0$, only $n-2$ of these equations will be independent. As before we can normalize by setting $z_{n}=0, z_{n-1}=1$. When $n=4$ we have two polynomial equations for $z_{1}, z_{2}$ :

$$
\begin{aligned}
& G_{2}\left(z_{1}, z_{2}, 1,0\right)=\left(\Gamma_{2}+\Gamma_{4}\right)\left(-z_{2} z_{1}^{2}+z_{1}^{2}+z_{2}^{2} z_{1}-z_{2} z_{1}\right) \\
&+\Gamma_{1}\left(z_{2}^{3}-z_{2}^{2}\right)+\Gamma_{3}\left(z_{1} z_{2}^{3}-z_{1}^{2} z_{2}^{2}\right)=0 . \\
& G_{3}\left(z_{1}, z_{2}, 1,0\right)=\Gamma_{2}\left(z_{1}-z_{1}^{2}\right)+\Gamma_{1}\left(z_{2}-z_{2}^{2}\right) \\
&+\left(\Gamma_{3}+\Gamma_{4}\right)\left(z_{2}^{2} z_{1}^{2}-z_{2} z_{1}^{2}-z_{2}^{2} z_{1}+z_{2} z_{1}\right)=0 .
\end{aligned}
$$


Their resultant is $r\left(z_{1}\right)=z_{1}^{3}\left(1-z_{1}\right) P_{6}\left(z_{1}\right)$ where $P_{6}\left(z_{1}\right)$ is a complicated 6 -th degree polynomial

$$
P_{6}\left(z_{1}\right)=C_{6} z_{1}^{6}+\ldots+C_{0}
$$

where $C_{i}\left(\Gamma_{1}, \ldots, \Gamma_{4}\right)$ are polynomials in the vorticities. For example, after using $\Gamma=0$ to simplify, we have:

$$
C_{6}=-\Gamma_{1}^{2}\left(\Gamma_{3}+\Gamma_{4}\right)\left(\Gamma_{2} \Gamma_{3}+\Gamma_{2} \Gamma_{4}+\Gamma_{3} \Gamma_{4}\right) .
$$

Although the individual coefficients may vanish for certain choices of the vorticities, they never vanish simultaneously when $\Gamma_{i} \neq 0$. To show this we appended a normalization condition $\Gamma_{1} \Gamma_{2} \Gamma_{3} \Gamma_{4}=1$ to the list of coefficients and found a Gröbner basis for the resulting polynomial ideal. The basis consists of the constant polynomial 1 which shows that, under the normalization condition, the coefficients cannot all be zero.

The following lemma allows us to conclude that there can never be more than 6 rigidly translating solutions.

Lemma 2. Let $f\left(z_{1}, z_{2}\right), g\left(z_{1}, z_{2}\right)$ be polynomials such that the resultant $r\left(z_{1}\right)=$ $\operatorname{Res}\left(f, g, z_{2}\right)$ is not identically zero. If $\zeta$ is a root of $r\left(z_{1}\right)$ of multiplicity $\mu$, then there are at most $\mu$ complex solutions $z_{2}$ to the system of equations $f\left(\zeta, z_{2}\right)=g\left(\zeta, z_{2}\right)=0$.

We will omit the proof since this is a variant of a classical result about intersections of algebraic curves which will now be described. The equations $f=0$ and $g=0$ define affine curves $\mathcal{C}$ and $\mathcal{D}$ in $\mathbf{C}^{2}$. The common solutions of the form $\left(\zeta, z_{2}\right)$ are just the points of $\mathcal{C} \cap \mathcal{D}$ which project to $\zeta$. The homogenized equations $F\left(z_{0}, z_{1}, z_{2}\right)=0$ and $G\left(z_{0}, z_{1}, z_{2}\right)=0$ define curves in $\mathbf{C P}^{2}$ which will also be denoted by $\mathcal{C}$ and $\mathcal{D}$. A classical theorem about algebraic curves shows that if the point at infinity $\left[z_{0}, z_{1}, z_{2}\right]=[0,0,1]$ is not in $\mathcal{C} \cap \mathcal{D}$, then the multiplicity $\mu$ is exactly the sum of the intersection multiplicities of the curves at points of the form $\left[1, \zeta, z_{2}\right]$ [29, Theorem 7.5]. It turns out that if $[0,0,1] \in \mathcal{C} \cap \mathcal{D}$, there can be an additional, nonnegative contribution to $\mu$ which does not arise from points of intersection. But in all cases, $\mu$ is an upper bound for the number of intersection points which project to $\zeta$.

Applying the lemma to the polynomials defining rigid translations shows that there are at most 6 solutions which project to roots of the polynomial $P_{6}\left(z_{1}\right)$. Any other solutions must project to $z_{1}=0,1$ and so lie in $\Delta$. This proves Theorem 1(ii).

For certain vorticities, there will be fewer than 6 rigidly translating solutions. For example, if $\Gamma_{3}+\Gamma_{4}=0$ or $\Gamma_{2} \Gamma_{3}+\Gamma_{2} \Gamma_{4}+\Gamma_{3} \Gamma_{4}=0$, the leading coefficient $C_{6}=0$ and there will be fewer than 6 roots. In addition, we have

$P_{6}(0)=\Gamma_{1}^{2}\left(\Gamma_{2}+\Gamma_{3}\right)\left(\Gamma_{1} \Gamma_{2}+\Gamma_{1} \Gamma_{4}+\Gamma_{2} \Gamma_{4}\right), \quad P_{6}(1)=\Gamma_{1}^{2}\left(\Gamma_{2}+\Gamma_{4}\right)\left(\Gamma_{1} \Gamma_{2}+\Gamma_{1} \Gamma_{3}+\Gamma_{2} \Gamma_{3}\right)$

which shows that for certain vorticities, some of the 6 solutions project to $z_{1}=0,1$ and so lie in $\Delta$.

\section{Collinear Relative equilibria}

Collinear relative equilibria can also be handled in a comparatively simple way. The equations for relative equilibria are $V_{i}=I \lambda\left(z_{i}-c\right)$ where $\lambda \neq 0 \in \mathbf{R}$ and $c \in \mathbf{C}$ are constants. Multiplying by $\Gamma_{i}$ and summing gives the relation

$$
\lambda(C-\Gamma c)=0
$$


where $C=\sum_{i=1}^{n} \Gamma_{i} z_{i}$. If $\Gamma \neq 0$ we can solve for the center of rotation $c$, but we do not want to make this assumption. We get another relation by multiplying $\Gamma_{i} \bar{z}_{i}$ and summing:

$$
L=\lambda S,
$$

where $S=\sum_{i=1}^{n} \Gamma_{i}\left|z_{i}-c\right|^{2}$ is the moment of inertia with respect to the rotation center.

Now the relative equilibrium equations involve both the positions $z_{i}$ and their conjugates. However, for collinear relative equilibria we may assume that all the positions are real, so $z_{i}=\bar{z}_{i}$. Following O'Neil [26] we can replace $V_{i}$ by $\bar{V}_{i}$ and study the equations $\bar{V}_{i}=I \lambda\left(z_{i}-c\right)$. We continue to view the $z_{i}$ as complex variables, but only the real solutions will represent collinear relative equilibria.

Since the equations are not independent, we may subtract the $n$-th equation from the others to obtain $\bar{V}_{i}-\bar{V}_{n}=I \lambda\left(z_{i}-z_{n}\right)$. Clearing denominators leads to polynomial equations $H_{i}\left(z_{1}, \ldots, z_{n}\right)=0, i=1, \ldots, n-1$, where

$$
H_{i}=G_{i}+\lambda\left(z_{i}-z_{n}\right) \prod_{k \neq i, n}\left(z_{i}-z_{k}\right) \prod_{k \neq n}\left(z_{n}-z_{k}\right)
$$

and $G_{i}$ are as in (6). The terms involving $\lambda$ have degree $2 n-2$ in the $z_{i}$. We are still free to normalize by setting $z_{n-1}=1$ and $z_{n}=0$. Then we have $n-1$ nonhomogeneous equations for $z_{1}, \ldots, z_{n-2}, \lambda$.

When $n=4$ we have

$$
\begin{aligned}
H_{1} & =\Gamma_{2}\left(z_{1}^{3}-z_{1}^{2}\right)+\left(\Gamma_{1}+\Gamma_{4}\right)\left(z_{2} z_{1}^{2}-z_{2}^{2} z_{1}-z_{2} z_{1}+z_{2}^{2}\right)+\Gamma_{3}\left(z_{1}^{3} z_{2}-z_{1}^{2} z_{2}^{2}\right)=0, \\
H_{2} & =\left(\Gamma_{2}+\Gamma_{4}\right)\left(-z_{2} z_{1}^{2}+z_{1}^{2}+z_{2}^{2} z_{1}-z_{2} z_{1}\right)+\Gamma_{1}\left(z_{2}^{3}-z_{2}^{2}\right)+\Gamma_{3}\left(z_{1} z_{2}^{3}-z_{1}^{2} z_{2}^{2}\right)=0, \\
H_{3} & =\Gamma_{2}\left(z_{1}-z_{1}^{2}\right)+\Gamma_{1}\left(z_{2}-z_{2}^{2}\right)+\Gamma_{3}\left(z_{2}^{2} z_{1}^{2}-z_{2} z_{1}^{2}-z_{2}^{2} z_{1}+z_{2} z_{1}\right) \\
& +\Gamma_{4}\left(-z_{2} z_{1}^{2}+z_{1}^{2}+z_{2}^{2} z_{1}-z_{2} z_{1}\right)=0 .
\end{aligned}
$$

Using the third equation to eliminate $\lambda$ from the other two gives two equations for $z_{1}, z_{2}$ :

$$
z_{1} z_{2}\left(z_{1}-1\right) K_{2}=z_{1} z_{2}\left(z_{2}-1\right) K_{3}=0
$$

where

$$
\begin{aligned}
K_{2} & =\Gamma_{1}\left(2 z_{1}-1\right)\left(z_{1}-z_{2}\right)\left(z_{2}-1\right) z_{2}+\Gamma_{2} z_{1}^{2}\left(z_{1}-1\right)^{2} \\
& -\Gamma_{3}\left(z_{1}-2\right) z_{1}^{2}\left(z_{1}-z_{2}\right)\left(z_{2}-1\right) z_{2}-\Gamma_{4}\left(z_{1}+1\right)\left(z_{1}-z_{2}\right)\left(z_{2}-1\right) z_{2}\left(z_{1}-1\right)^{2}, \\
K_{3} & =\Gamma_{1} z_{2}^{2}\left(z_{2}-1\right)^{2}-\Gamma_{2}\left(z_{1}-1\right) z_{1}\left(z_{1}-z_{2}\right)\left(2 z_{2}-1\right) \\
& +\Gamma_{3}\left(z_{1}-1\right) z_{1}\left(z_{1}-z_{2}\right)\left(z_{2}-2\right) z_{2}^{2}+\Gamma_{4}\left(z_{1}-1\right) z_{1}\left(z_{1}-z_{2}\right)\left(z_{2}+1\right)\left(z_{2}-1\right)^{2} .
\end{aligned}
$$

The resultant of $K_{2}$ and $K_{3}$ with respect to $z_{2}$ is of the form

$$
r\left(x_{1}\right)=z_{1}^{5}\left(z_{1}-1\right)^{5} P_{12}\left(z_{1}\right),
$$

where $P_{12}\left(z_{1}\right)$ is a polynomial of degree 12 with coefficients $C_{i}\left(\Gamma_{1}, \ldots, \Gamma_{4}\right)$ depending on the vorticities. As before, a Gröbner basis of the ideal generated by the $C_{i}$ together with the normalization condition $\Gamma_{1} \Gamma_{2} \Gamma_{3} \Gamma_{4}=1$ consists of the constant polynomial 1 , so the resultant is never identically zero. Then Lemma 2 gives Theorem 1(iii). 


\section{Strictly Planar Relative EQUilibria}

In this case the defining equations involve both the complex variables $z_{i}$ and their conjugates. It is necessary to find some other way to put the equations into polynomial form.

5.1. Strictly planar relative equilibria with $\Gamma \neq 0$. When $\Gamma \neq 0$ there is a particularly elegant way to do this due to Albouy and Chenciner [1, 13. The result is the system

$$
\sum_{k=1}^{n} \Gamma_{k}\left[S_{i k}\left(r_{j k}^{2}-r_{i k}^{2}-r_{i j}^{2}\right)+S_{j k}\left(r_{i k}^{2}-r_{j k}^{2}-r_{i j}^{2}\right)\right]=0
$$

for $1 \leq i<j \leq n$, where $\lambda=\Gamma \lambda^{\prime}$ and the $S_{i j}$ are given by

$$
S_{i j}=\frac{1}{r_{i j}^{2}}+\lambda^{\prime} \quad(i \neq j), \quad S_{i i}=0 .
$$

This is a polynomial system after clearing the denominators in the $S_{i j}$ terms. We will also impose the normalization $\lambda^{\prime}=-1$.

We supplemented the Albouy-Chenciner equations with some additional constraints that are specialized to the strictly planar case (the Dziobek equations). The Dziobek equations [10, 2] for vortices, in terms of $s_{i j}=r_{i j}^{2}$, are

$$
\left(s_{i j}^{-1}-1\right)\left(s_{k l}^{-1}-1\right)=\left(s_{i k}^{-1}-1\right)\left(s_{j l}^{-1}-1\right)
$$

for $\{i, j, k, l\}=\{1,2,3,4\}$.

We now apply the methods employed in 13 to our polynomial system. The approach is based on the BKK theory which is concerned with finding solutions of algebraic equations with all variables nonzero complex numbers. This is particularly appropriate here since the variables are the six squared mutual distances $s_{i j}$.

Here is a sketch of the main idea. If there were infinitely many such solutions to our system, then there would be at least an algebraic curve of solutions. This curve of solutions can be expanded in a Puiseux series, i.e., each variable is given by a series of the form

$$
\sum_{i=i_{0}}^{\infty} a_{i} t^{\frac{i}{q}} .
$$

The initial term must be nonzero $\left(a_{0} \neq 0\right)$.

Consider the general setting of a system of $m$ polynomial equations in $n$ complex variables:

$$
f_{i}\left(x_{1}, \ldots, x_{n}\right)=\sum_{k} c_{k} x_{1}^{k_{1}} \ldots x_{n}^{k_{n}}=0, \quad i=1, \ldots, m,
$$

where the exponent vector $k=\left(k_{1}, \ldots, k_{n}\right)$ runs over a finite set $S_{i} \subset \mathbf{Z}^{n}$ (called the support of $f_{i}$ ). We seek solutions in the algebraic torus $T=(\mathbf{C} \backslash 0)^{n}$.

A Puiseux series solution would take the form $x(t)=\left(x_{1}(t), \ldots, x_{n}(t)\right)$ where $x_{j}(t)=a_{j} t^{\alpha_{j}}+\ldots, j=1, \ldots, n$. Such a solution will be said to have order $\alpha=\left(\alpha_{1}, \ldots, \alpha_{n}\right)$ ( $\alpha$ is the rational vector of exponents of the leading terms of the series). Then to prove that $V$ is finite, it suffices to show that for every nonzero rational vector $\alpha$ there is no Puiseux series solution of order $\alpha$. 
Substituion of these series into the system of equations and looking at the leading terms leads to a reduced system of equations for the leading coefficients. A reduced system for a vector $\alpha$ consists of

$$
f_{i \alpha}\left(a_{1}, \ldots, a_{n}\right):=\sum_{\alpha \cdot k=\mu_{i}} c_{k} x_{1}^{k_{1}} \ldots x_{n}^{k_{n}}=0, \quad i=1, \ldots, m,
$$

where $\mu_{i}=\min _{l \in S_{i}} \alpha \cdot l$. There is a nice geometric interpretation of the reduction process. The Newton polytope of $f_{i}$ is defined as the convex hull of its support. Then the exponent vectors $l$ which minimize $\alpha \cdot l$ lie on a face of the Newton polytope for which $\alpha$ is an inward-pointing normal vector.

The following proposition allows us to ignore some of the directions in exponent space:

Proposition 1. Let $H$ be the half-space $c \cdot \alpha \geq 0$ where $c=\left(c_{1}, \ldots, c_{n}\right)$ is an arbitrary, nonzero integer vector. If system (13) has no Puiseux series solutions of order $\alpha$ for all $\alpha \in H$, then it has finitely many solutions in $\mathbf{T}$.

Our main tool is the following:

Proposition 2. Let $\alpha$ be a nonzero rational vector. If the reduced system (14) has no solutions in $\mathbf{T}$, then there does not exist a Puiseux series solution of the full system (13) of order $\alpha$.

We now apply the above techniques to our equations. There are six variables $s_{i j}=r_{i j}^{2}$. The six Albouy-Chenciner equations are rational functions in these variables. Since we are looking for solutions with $s_{i j} \neq 0$ we can clear denominators to obtain polynomial equations. Similarly, the three Dziobek equations are equivalent to polynomial equations in the $s_{i j}$. Here is one of the Albouy-Chenciner equations expanded out:

$$
\begin{gathered}
-2 \Gamma s_{12} s_{13} s_{14} s_{23} s_{24}+\Gamma_{4} s_{12} s_{13} s_{14} s_{23}-\Gamma_{4} s_{13} s_{14}^{2} s_{23}+\Gamma_{3} s_{12} s_{13} s_{14} s_{24} \\
-\Gamma_{3} s_{13}^{2} s_{14} s_{24}+\Gamma_{4} s_{12} s_{13} s_{23} s_{24}+\Gamma_{3} s_{12} s_{14} s_{23} s_{24} \\
+2 \Gamma s_{13} s_{14} s_{23} s_{24}-\Gamma_{3} s_{14} s_{23}^{2} s_{24}-\Gamma_{4} s_{13} s_{23} s_{24}^{2}=0 .
\end{gathered}
$$

The others are similar. Since there are ten distinct monomials, the corresponding Newton polytope in $\mathbf{R}^{6}$ has ten vertices. For example, if we order our six variables $s=\left(s_{12}, s_{13}, s_{14}, s_{23}, s_{24}, s_{34}\right)$, then the vector of exponents of the first term of the polynomial above is $k=(1,1,1,1,1,0) \in \mathbf{R}^{6}$ and this point will be one of the vertices of the Newton polytope.

Recall that we are adding the Dziobek equations (Eq. (11)) to our system. Once denominators are cleared these appear as follows:

$$
\begin{aligned}
& s_{14} s_{23}-s_{12} s_{34}-s_{12} s_{14} s_{23}+s_{12} s_{14} s_{34}+s_{12} s_{23} s_{34}-s_{14} s_{23} s_{34}=0, \\
& s_{13} s_{24}-s_{12} s_{34}-s_{12} s_{13} s_{24}+s_{12} s_{13} s_{34}+s_{12} s_{24} s_{34}-s_{13} s_{24} s_{34}=0, \\
& s_{14} s_{23}-s_{13} s_{24}-s_{13} s_{14} s_{23}+s_{13} s_{14} s_{24}+s_{13} s_{23} s_{24}-s_{14} s_{23} s_{24}=0 .
\end{aligned}
$$

Each of their Newton polytopes has six vertices. The Minkowski sum polytope, $P \subset \mathbf{R}^{6}$, of these nine Newton polytopes, was computed by adding the vertices and then finding a minimal set of vertices and facets with the program Porta 1.3.2 [8]. It turns out that $P$ has 4608 vertices and 442 facets. The Minkowski sum of the Newton polytopes of the Albouy-Chenciner equations alone has 943 vertices and 129 facets. 
TABLE 1. Nontrivial facet normal representatives for the vortex Minkowski sum polytope

\begin{tabular}{|c|c|c|c|c|c|}
\hline 1. & ( 1 , & 1 , & 1 & & 1, \\
\hline 2. & ( 0 , & 1 , & 1 & & \\
\hline 3. & ( 0 , & 0 , & 0 & & 1, \\
\hline 4. & $(0$, & 0 , & 1 & & 0 , \\
\hline 5. & $(0$, & 0 , & 0 & & 0 , \\
\hline 6. & $(-1$, & 0 , & 0 & & 0 \\
\hline 7. & $(-1)$ & -1 & -1 & & 0 , \\
\hline 8. & $(-1$, & -1 & 0 & & 1, \\
\hline 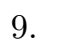 & $(-1$, & -1 & 0 & & \\
\hline
\end{tabular}

The second step of our procedure is to analyze the reduced systems corresponding to the various faces of $P$. The simplest faces are those whose reduced systems contain a monomial, i.e., one or more of the reduced equations consists of a single term. We will refer to such a face or reduced system as trivial. A trivial reduced system can have a solution with all variables nonzero only if the coefficient of the monomial vanishes. Since the monomial is one of the terms of the original equations, all possible trivial reduced systems will have no nonzero solutions provided that all of the original coefficients are nonzero. For the equations above, this amounts to assuming that the vorticities $\Gamma_{i} \neq 0$ and that the sum of the vorticities $\Gamma \neq 0$. These are standing assumptions in this subsection.

Using Mathematica 34 we found that all but 35 of the 442 facets of the polytope $P$ are trivial. Up to the $S_{4}$ symmetry of our system, there are 9 representatives of the 35 nontrivial facets. The inward-pointing normals to these facets are listed in Table 1.

For example, for the facet normal 6 , we obtain the reduced equations by selecting from each of the nine polynomials $f_{i}$ of our system, those monomials whose exponent vectors minimize $\alpha \cdot k=-k_{1}-k_{6}$ among all the monomials in $f_{i}$. After cancelling out factors which are powers of the variables $s_{i j}$, the result is the following system of nine reduced equations $f_{i \alpha}$ :

$$
\begin{aligned}
& s_{14}+s_{23}-1=s_{13}+s_{24}-1=0, \\
& \Gamma_{1} s_{12} s_{24}+\Gamma_{4} s_{13} s_{34}=\Gamma_{1} s_{12} s_{23}+\Gamma_{3} s_{14} s_{34}=0, \\
& \Gamma_{2} s_{12} s_{14}+\Gamma_{4} s_{23} s_{34}=\Gamma_{2} s_{12} s_{13}+\Gamma_{3} s_{24} s_{34}=0, \\
& s_{14} s_{23}-s_{13} s_{24}-s_{13} s_{14} s_{23}+s_{13} s_{24} s_{23}-s_{14} s_{24} s_{23}+s_{13} s_{14} s_{24}=0, \\
& \Gamma_{1} s_{13} s_{24} s_{23}+\Gamma_{1} s_{14} s_{24} s_{23}+\Gamma_{2} s_{13} s_{14} s_{23}+\Gamma_{2} s_{13} s_{14} s_{24}-2 \Gamma s_{13} s_{14} s_{24} s_{23}=0, \\
& \Gamma_{4} s_{13} s_{14} s_{23}+\Gamma_{4} s_{13} s_{24} s_{23}+\Gamma_{3} s_{14} s_{24} s_{23}+\Gamma_{3} s_{13} s_{14} s_{24}-2 \Gamma s_{13} s_{14} s_{24} s_{23}=0 .
\end{aligned}
$$

All such systems of reduced equations have a certain homogeneity. In this case, the monomials which appear in each equation have exponent vectors $k$ with the same value of $-k_{1}-k_{6}$. It follows that if $s=\left(s_{12}, s_{13}, s_{14}, s_{23}, s_{24}, s_{34}\right) \in \mathbf{T}$ is any solution, then for any $t \in \mathbf{C} \backslash 0$, we get another solution $\left(t^{-1} s_{12}, s_{13}, s_{14}, s_{23}, s_{24}, t^{-1} s_{34}\right)$. In general, for the reduced equations $f_{i \alpha}$ we can rescale solutions via

$$
\left(t^{\alpha_{1}} s_{12}, t^{\alpha_{2}} s_{13}, t^{\alpha_{3}} s_{14}, t^{\alpha_{4}} s_{23}, t^{\alpha_{5}} s_{24}, t^{\alpha_{6}} s_{34}\right) .
$$


Using this homogeneity, we can arrange to have the following normalization condition hold:

$$
s_{12} s_{13} s_{14} s_{23} s_{24} s_{34}=1 \text {. }
$$

To see this, note that under the rescaling above, the product of the $s_{i j}$ changes by a factor for $t^{|\alpha|}$, where $|\alpha|=\alpha_{1}+\ldots+\alpha_{6}$. As long as $|\alpha| \neq 0$ and $s_{i j} \neq 0$, we can rescale to make the product be 1 .

Now the goal is to show that the reduced systems have no solutions $s \in \mathbf{T}$. To do this we append the normalization condition (15) to get ten equations and then use the Gröbner basis method to eliminate the variables $s_{i j}$. The equations define an ideal in the polynomial ring with variables $s_{i j}$ and $\Gamma_{i}$. If this ideal contains polynomials in the vorticity variables alone, then these polynomials give necessary conditions on the vorticities for the existence of values $s_{i j} \in \mathbf{C}$, making the equations valid. In particular if these mass polynomials are not zero for a given choice of $\Gamma_{i}$, then, for those $\Gamma_{i}$, the reduced equations have no solutions $s \in \mathbf{T}$ as required. Such polynomials in the vorticities alone can be found by computing a Gröbner basis for the ideal with an appropriately chosen monomial ordering (for an introduction to Gröbner basis theory, see [9]). Our Gröbner basis calculations were carried out using Mathematica and checked using Macaulay 2 [1] and/or MAGMA 21.

Using Proposition 1 we can restrict attention to facets and lower-dimensional faces having an inward normal vector such that $c \cdot \alpha \geq 0$ where $c=-(1,1,1,1,1,1)$. This means that we only need to check facets $6-9$ in the table. For facets 8 and 9 the Gröbner basis computation shows that the polynomial ideal in the last paragraph is generated by the constant polynomial 1 . This means that the system of equations has no solutions. Facets 6 and 7 are more complicated and will be treated in the next subsection.

5.1.1. Facets 6 and $\%$. For the planar four-vortex system there are only two reduced systems which have nonzero solutions for real $\Gamma_{i} \neq 0$. The first is that of the exponent vector 6 from Table 1 i.e., $\alpha=(-1,0,0,0,0,-1)$. A Gröbner basis for the normalized reduced equations consists of the polynomial $\Gamma_{1} \Gamma_{2}-\Gamma_{3} \Gamma_{4}$. Thus if this polynomial vanishes, the reduced system is solvable. This means that we can find the leading terms of a possible Puiseux series solution. However, as in [13, if we attempt to continue the series we obtain a contradiction at the next order of the expansion.

The other reduced system with nonzero solutions is that of the exponent vector 7 from Table 1 i.e., $\alpha=(-1,-1,-1,0,0,0)$. From the Gröbner basis described above we find the following requirements for the vorticities:

$$
\begin{array}{r}
\Gamma_{2}^{2}+\Gamma_{3}^{2}+\Gamma_{4}^{2}-\Gamma_{2} \Gamma_{3}-\Gamma_{3} \Gamma_{4}-\Gamma_{2} \Gamma_{4}=0 \\
3 \Gamma_{1}+\Gamma_{2}+\Gamma_{3}+\Gamma_{4}=0 .
\end{array}
$$

It is not hard to see that the only real, nonzero vorticities satisfying both of the polynomials above are of the form $\left(\Gamma_{1}, \Gamma_{2}, \Gamma_{3}, \Gamma_{4}\right)=k(-1,1,1,1)$ where $k \neq 0$. This particular case can be solved directly by computing a Gröbner basis (done with the program MAGMA [21]). For its possible independent interest we include 
the Gröbner basis below:

$$
\begin{aligned}
& \left\{-316+369 s_{12}+1813 s_{34}-7234 s_{34}^{2}-9694 s_{34}^{3}+33766 s_{34}^{4}-11660 s_{34}^{5}-18388 s_{34}^{6}\right. \\
& +13592 s_{34}^{7}-2248 s_{34}^{8}=0, \\
& 842+369 s_{13}+369 s_{24}-5375 s_{34}-738 s_{24} s_{34}-4789 s_{34}^{2}+27806 s_{34}^{3}+1476 s_{24} s_{34}^{3} \\
& -14588 s_{34}^{4}-18452 s_{34}^{5}+13712 s_{34}^{6}-64 s_{34}^{7}-568 s_{34}^{8}=0, \\
& 785+738 s_{14}+738 s_{24}-16703 s_{34}-706 s_{34}^{2}+77276 s_{34}^{3}-81968 s_{34}^{4}-14768 s_{34}^{5} \\
& +57800 s_{34}^{6}-26824 s_{34}^{7}+3632 s_{34}^{8}=0 \text {, } \\
& -89+246 s_{23}-246 s_{24}-3439 s_{34}+492 s_{24} s_{34}+7780 s_{34}^{2}+13684 s_{34}^{3}-984 s_{24} s_{34}^{3} \\
& -40108 s_{34}^{4}+15152 s_{34}^{5}+22384 s_{34}^{6}-17960 s_{34}^{7}+3088 s_{34}^{8}=0 \text {, } \\
& -3019-2214 s_{24}+738 s_{24}^{2}+6397 s_{34}-2952 s_{24} s_{34}+19268 s_{34}^{2}+1476 s_{24} s_{34}^{2} \\
& -26734 s_{34}^{3}+1476 s_{24} s_{34}^{3}-7136 s_{34}^{4}+17692 s_{34}^{5}-4576 s_{34}^{6}-640 s_{34}^{7}+224 s_{34}^{8}=0 \text {, } \\
& \left(8+9 s_{24}-41 s_{34}+14 s_{34}^{2}+14 s_{34}^{3}-4 s_{34}^{4}\right)\left(1-6 s_{34}^{2}+4 s_{34}^{4}\right)=0, \\
& \left.\left(-1+s_{34}\right)\left(1-6 s_{34}^{2}+4 s_{34}^{4}\right)\left(-1-16 s_{34}+38 s_{34}^{2}-24 s_{34}^{3}+4 s_{34}^{4}\right)=0\right\} \text {. }
\end{aligned}
$$

From the Gröbner basis it is easy to compute that besides the spatial regular tetrahedron $\left(s_{12}, s_{13}, s_{14}, s_{23}, s_{24}, s_{34}\right)=(1,1,1,1,1,1)$ there are 12 other solutions, all of which possess a symmetry of the form $s_{1 i}=s_{i j}, s_{1 k}=s_{j k}$ for distinct $(i, j, k)=(1,2,3)$. Only six of these are entirely positive and real. Note that these do not include the equilibrium with $s_{12}=s_{13}=s_{14} \neq s_{23}=s_{34}=s_{24}$ since we normalized the rotation rate.

5.1.2. Lower-dimensional faces. We also need to examine the reduced systems arising from lower-dimensional faces of the Minkowski sum polytope. These can be found by intersecting adjacent facets. Such a face determines infinitely many inward normal vectors obtained by convex combinations of the inward normals of the intersecting facets. However, all these normal vectors $\alpha$ determine the same reduced system. If this reduced system has no solutions, then all of the corresponding normal vectors $\alpha$ are eliminated as possible orders of Puiseux series solutions.

In testing these lower-dimensional faces, we again exploit permutation symmetry to cut down the number of cases which need to be tested. Also, we only have to test faces which have at least one normal vector $\alpha$ satisfying $c \cdot \alpha \geq 0$.

As for the facets, it turns out that there are three kinds of reduced systems arising from these faces. For most of the faces, the corresponding reduced system is trivial. For many of the remaining faces, the reduced system is nontrivial but the Gröbner basis computation shows that it nevertheless has no solutions. Finally, there are a few lower-dimensional faces for which the reduced system is indeed solvable.

For example, among the 35 nontrivial facets are two with internal normal vectors $\alpha^{\prime}=(0,0,0,1,1,1)$ and $\alpha^{\prime \prime}=(-1,-1,-1,0,0,0)$. These intersect in a face whose internal normal could be chosen as $\alpha=k \alpha^{\prime}+l \alpha^{\prime \prime}$ for any $k, l>0$. To get a rational normal vector, we just need to take rational $k, l$. As long as $l \geq k$, we will have $c \cdot \alpha \geq 0$. A Gröbner basis for the normalized reduced equations is the polynomial $\Gamma_{2}+\Gamma_{3}+\Gamma_{4}$. It follows that if this quantity is nonzero, the reduced system has no solutions. 
If $\Gamma_{2}+\Gamma_{3}+\Gamma_{4}=0$, the reduced system is solvable and it is necessary to look at higher order terms in the Puiseux series. Unfortunately, we were unable to show that such a series was impossible. One difficulty is that, unlike the case of facet 6 in the last subsection, one does not even know the exponent vector $\alpha$ of the leading terms unambiguously.

In Theorem 1 we have assumed that $\Gamma_{i}+\Gamma_{j}+\Gamma_{k} \neq 0$ to eliminate this case and other similar cases obtained from permutation symmetry. Other combinations of facets yield faces whose reduced systems are solvable when $\Gamma_{i}+\Gamma_{j}=0$ and so we have also eliminated these by hypothesis. No other polynomials in the vorticities arise. Difficulties with such lower-dimensional faces were avoided in our study 13 of the Newtonian four-body problem because the masses are positive.

5.1.3. Upper bounds. To obtain an upper bound for the strictly planar relative equilibria with $\Gamma \neq 0$, we can use an argument identical to that in [13]. Namely we construct an auxiliary system of ten equations and ten variables and use the mixed volume of its Newton polytope for our bound.

Consider the 11 equations

$$
\begin{aligned}
& f_{0}=\Gamma_{1} z_{1}+\Gamma_{2} z_{2}+\Gamma_{3} z_{3}+\Gamma_{4} z_{4}=0, \\
& f_{1}=\Gamma_{2} z_{2} s_{12}+\Gamma_{3} z_{3} s_{13}+\Gamma_{4} z_{4} s_{14}+k=0, \\
& f_{2}=\Gamma_{1} z_{1} s_{12}+\Gamma_{3} z_{3} s_{23}+\Gamma_{4} z_{4} s_{24}+k=0, \\
& f_{3}=\Gamma_{1} z_{1} s_{13}+\Gamma_{2} z_{2} s_{23}+\Gamma_{4} z_{4} s_{34}+k=0, \\
& f_{4}=\Gamma_{1} z_{1} s_{14}+\Gamma_{2} z_{2} s_{24}+\Gamma_{3} z_{3} s_{34}+k=0, \\
& S_{i j}=z_{i} z_{j} \quad 1 \leq i<j \leq 4,
\end{aligned}
$$

in 11 variables $z_{1}, \ldots, z_{4}, s_{12}, \ldots, s_{34}, k$, where

$$
S_{i j}=\frac{1}{s_{i j}}+\lambda^{\prime}=\frac{1}{s_{i j}}-1
$$

As described in [13], every strictly planar relative equilibrium determines a solution of (16) where each $z_{i}$ is proportional to the area of the triangles formed by the vortices other than $\Gamma_{i}$ and the $s_{i j}$ are the squared mutual distances. For such a solution, all of the variables $z_{i}, s_{i j}$ must be nonzero. If, for example, $z_{4}=0$, then the vortices $\Gamma_{1}, \Gamma_{2}, \Gamma_{3}$ would be collinear because they span a triangle of zero area. But the equations $S_{i 4}=0$ imply that $s_{i 4}=1$, so $\Gamma_{1}, \Gamma_{2}, \Gamma_{3}$ also lie on a unit circle around $\Gamma_{4}$. Clearly this is geometrically impossible.

Now eliminate $k$ by replacing $f_{1}, \ldots, f_{4}$ with the differences $f_{1}-f_{4}, f_{2}-f_{4}, f_{3}-f_{4}$ to obtain 10 equations in 10 unknowns. It suffices to find an upper bound for the number of solutions of this system with all variables nonzero. First it will be shown that the number of solutions is finite by showing that there is a 2 to 1 mapping from solutions of these equations to solutions of the Albouy-Chenciner and Dziobek equations.

First it is clear that the equations $S_{i j}=z_{i} z_{j}$ imply Dziobek's equations (11). Now the Albouy-Chenciner equation (9) with $(i, j)=(1,2)$ and $S_{i j}=z_{i} z_{j}$ reads:

$$
\begin{aligned}
-2\left(\Gamma_{1}+\Gamma_{2}\right) z_{1} z_{2} s_{12} & +\Gamma_{3} z_{1} z_{3}\left(s_{23}-s_{13}-s_{12}\right)+\Gamma_{3} z_{2} z_{3}\left(s_{13}-s_{23}-s_{12}\right) \\
& +\Gamma_{4} z_{1} z_{4}\left(s_{24}-s_{14}-s_{12}\right)+\Gamma_{4} z_{2} z_{4}\left(s_{14}-s_{24}-s_{12}\right) .
\end{aligned}
$$

This agrees with the expression

$$
-\left(z_{1}+z_{2}\right) s_{12} f_{0}+\left(z_{2}-z_{1}\right)\left(f_{1}-f_{2}\right)
$$


The other Albouy-Chenciner equations can be written in a similar way. Hence our system of 10 equations implies the Albouy-Chenciner and Dziobek equations.

On the other hand, given any solution $s_{i j}$ of the Albouy-Chenciner and Dziobek equations, it is easy to see that the equations

$$
z_{i} z_{j}=\frac{1}{s_{i j}}-1
$$

determine the $z_{i}$ up to a common factor of \pm 1 , hence the 2 to 1 mapping. Since the number of solutions of the Albouy-Chenciner and Dziobek equations has been shown to be finite, so is the number of solutions of the system of 10 equations.

Now for systems with equal numbers of equations and unknowns, the mixed volume of the Newton polytope provides an upper bound for the number of solutions with all variables nonzero, provided this number is finite. Our system of 10 equations has a Newton polytope with a mixed volume of 74 (computed using PHCpack [33]). This proves Theorem 1(v).

5.2. Strictly planar relative equilibria with $\Gamma=0$. Finally we consider planar relative equilibria with vanishing total vorticity, i.e., $\Gamma=0$. Equation (7) shows that $\lambda C=0$ where $C=\sum_{i=1}^{n} \Gamma_{i} z_{i}$. Since we want genuine relative equilibria rather than equilibria, we will look for solutions with $\lambda \neq 0$. Therefore we have $C=0$. This condition leads to some very simple equations in mutual distance coordinates, as shown in a recent study of the $n$-body problem with zero total mass by Celli 7 .

When $\Gamma=0$, the formula for $C$ is translation invariant, i.e., $C=\sum_{i=1}^{n} \Gamma_{i}\left(z_{i}-c\right)$ for any $c \in \mathbf{C}$. Celli observes that for configuations with $C=0$, the second moment $S=\sum_{i=1}^{n} \Gamma_{i}\left|z_{i}-c\right|^{2}$ is also independent of $c$. Taking $c$ to be the position of one of the four particles shows that the four quantities are equal:

$$
S_{i}=\Gamma_{j} s_{i j}+\Gamma_{k} s_{i k}+\Gamma_{l} s_{i l}, \quad\{i, j, k, l\}=\{1,2,3,4\},
$$

where $s_{i j}=\left|z_{i}-z_{j}\right|^{2}$. Taking differences gives three linear equations in the squared mutual distances, allowing us to eliminate $s_{12}, s_{13}, s_{23}$, for example. Celli shows that the remaining three squared distances can be used as coordinates on the set of noncollinear configurations with $C=0$.

These relations hold simply by virtue of the equations $\Gamma=C=0$. Celli also obtains simple equations for relative equilibria. In the vortex problem relative equilibria can be viewed as critical points of $U=\sum_{i<j} \Gamma_{i} \Gamma_{j} \log s_{i j}$, subject to the constraint $S=\sum_{i=1}^{n} \Gamma_{i}\left|z_{i}-c\right|^{2}=s_{0}$, where $c$ is the center of rotation of the relative equilibrium and $s_{0}$ is a constant. The discussion above shows that all of the critical points will automatically satisfy the constraints

$$
S_{1}=S_{2}=S_{3}=S_{4}=s_{0} .
$$

So one can impose these as independent constraints without changing the critical points. Using $s_{i j}$ as coordinates and introducing four Lagrange multipliers $\xi_{i}$ leads to six equations:

$$
\frac{\Gamma_{i} \Gamma_{j}}{s_{i j}}=\Gamma_{i} \xi_{j}+\Gamma_{j} \xi_{i}, \quad 1 \leq i<j \leq 4 .
$$

Eliminating the $\xi_{i}$ leads to the relations:

$$
\frac{1}{s_{12}}+\frac{1}{s_{34}}=\frac{1}{s_{13}}+\frac{1}{s_{24}}=\frac{1}{s_{14}}+\frac{1}{s_{23}} .
$$


We can impose the normalization $s_{34}=1$ and then use (18) to solve for $s_{12}$, $s_{13}, s_{23}$. Taking differences of the three quantities in (19) gives three equations involving $s_{14}, s_{24}$. After dropping constant factors and powers of the $\Gamma_{i}$, their pairwise resultants with respect to $s_{24}$ are of the form: $\left(\Gamma_{1}-\Gamma_{2}-\Gamma_{3}+\Gamma_{4}\right) P_{7}\left(s_{14}\right)$ and $\left(\Gamma_{1}+\Gamma_{2}-\Gamma_{3}-\Gamma_{4}\right) P_{7}\left(s_{14}\right)$, where $P_{7}\left(s_{14}\right)$ is a polynomial of degree 7 in $s_{14}$ with coefficients depending on the $\Gamma_{i}$.

The only vorticities which satisfy

$$
\Gamma_{1}+\Gamma_{2}+\Gamma_{3}+\Gamma_{4}=\Gamma_{1}-\Gamma_{2}-\Gamma_{3}+\Gamma_{4}=\Gamma_{1}+\Gamma_{2}-\Gamma_{3}-\Gamma_{4}=0
$$

are multiples of $(1,-1,1,-1)$. For these vorticities one can explicitly solve the equations and one finds two solutions for the squared mutual distances. Hence there are at most 4 relative equilibria in this case. In all other cases the relative equilibria must satisfy $P_{7}\left(s_{14}\right)=0$.

It is possible that the polynomial $P_{7}$ vanishes identically for some nonzero vorticities. To find them we first eliminated factors of the $\Gamma_{i}$ from the coefficients of $P_{7}$. Taking these eight polynomials together with $\Gamma_{1}+\Gamma_{2}+\Gamma_{3}+\Gamma_{4}$ and the normalization $\Gamma_{1} \Gamma_{2} \Gamma_{3} \Gamma_{4}=1$ gives a polynomial ideal which has the Gröbner basis:

$$
\Gamma_{1}+\Gamma_{4}, \quad \Gamma_{2}-\Gamma_{4}, \quad \Gamma_{3}+\Gamma_{4}, \quad \Gamma_{4}^{4}-1 .
$$

The only real solutions are multiples of $(1,-1,1,-1)$. In this case $P_{7}$ vanishes identically, but we have already seen that there are at most 4 relative equilibria.

In all other cases, Lemma 2 shows that we then have at most 7 values for the squared mutual distances $s_{i j}$ and therefore at most 14 relative equilibria. This completes the proof of Theorem 1 .

\section{REFERENCES}

1. A. Albouy and A. Chenciner, Le problème des $n$ corps et les distances mutuelles, Inv. Math. 131 (1998) 151-184. MR 1489897 (98m:70017)

2. A. Albouy, On a paper of Moeckel on central configurations, Reg. and Chao. Dyn. 8 (2003) 133-142. MR 1988854 (2004e:70011)

3. A. Albouy and R. Moeckel, The inverse problem for collinear central configurations, Celestial Mech. Dynam. Astronom. 77 (2001) 77-91. MR.1820352 (2002b:70017)

4. H. Aref and M. A. Stremler, Four-vortex motion with zero total circulation and impulse, Phys. Fluids 11, 12, 3704-3715. MR.1723520 (2000g:76018)

5. H. Aref, P. K. Newton, M. A. Stremler, T. Tokieda, and D. L. Vainchtein, Vortex Crystals, Advances In Applied Mechanics 39 (2003) 1-79.

6. D. N. Bernstein, The number of roots of a system of equations, Fun. Anal. Appl. 9 (1975) 183-185. MR0435072 (55:8034)

7. M. Celli, Sur les mouvements homographiques de $N$ corps associés à des masses de signe quelconque, le cas particulier où la somme des masses est nulle, et une application à la recherche de choréographies perverse, Thèse, Université Paris 7 (2005).

8. T. Christof and A. Loebel. PORTA: Polyhedron Representation Transformation Algorithm, Version 1.3.2. http://www.iwr.uni-heidelberg.de/ iwr/comopt/soft/PORTA/readme.html

9. D. Cox, J. Little, and D. O'Shea, Ideals, varieties, and algorithms. An introduction to computational algebraic geometry and commutative algebra, Springer-Verlag, New York, (1997). MR $1417938(97 \mathrm{~h}: 13024)$

10. O. Dziobek, Über einen merkwürdigen Fall des Vielkörperproblems, Astron. Nach. 152 (1900) 33-46.

11. D.R. Grayson and M. E. Sullivan, Macaulay 2, a software system for research in algebraic geometry and commutative algebra, http://www.math.uic.edu/Macaulay2/.

12. W. Gröbli, Specielle Probleme über die Bewegung geradliniger paralleler Wirfbelfäden, Vierteljahrschrift der naturforschenden Gesellschaft in Zürich 22 (1877) 37-81, 129-165. 
13. M. Hampton and R. Moeckel, Finiteness of relative equilibria of the four-body problem, Inventiones Mathematicae 163 (2006) 289-312. MR2207019 (2008c:70019)

14. M. Hampton and R. Moeckel, VortexCalculations.nb, Mathematica notebook available from http://www.math.umn.edu/ rick

15. H. Helmholtz, Uber Integrale der hydrodynamischen Gleichungen, Welche den Wirbelbewegungen entsprechen, Crelle's Journal für Mathematik, 55 (1858) 25-55. English translation by P. G. Tait, P.G., On the integrals of the hydrodynamical equations which express vortexmotion, Philosophical Magazine, (1867) 485-512.

16. Heron of Alexandria, Metrica, (circa 60).

17. A. G. Khovansky, Newton polyhedra and toric varieties, Fun. Anal. Appl. 11 (1977) 289-296.

18. G. R. Kirchhoff, Vorlesungen über Mathenatische Physik I, Teubner, Leipzig (1876).

19. A. G. Kushnirenko, Newton polytopes and the Bézout theorem, Fun. Anal. Appl. 10 (1976) 233-235.

20. C. C. MacDuffee, Theory of Matrices, Chelsea Publishing Co., New York (1946).

21. Computational Algebra Group, MAGMA, version 2.11.11, University of Sydney.

22. R. Moeckel, Chaotic dynamics near triple collision, Arch. Rational Mech. Anal. 107 (1989) no. 1, 37-69. MR.1000223 (90i:58167)

23. R. Moeckel, A Computer Assisted Proof of Saari's Conjecture for the Planar Three-Body Problem, Trans. AMS 357 (2005) no. 8, 3105-3117. MR2135737 (2005m:70054)

24. T. S. Motzkin, H. Raiffa, G. L. Thompson, and R. M. Thrall, The double description method, Annals of Mathematics Studies 28 (1953) 51-73. MR0060202 (15:638g)

25. E. A. Novikov, Dynamics and statistics of a system of vortices, Soviet Phys. JETP 41 (1975) 937-943.

26. K. A. O'Neil, Stationary configurations of point vortices, Trans. AMS 302 (1987) no. 2, 383-425. MR891628 (88d:76018)

27. K. A. O'Neil, Notes on relative equilibria of point vortices, personal communication, (2006).

28. G. Roberts, A continuum of relative equilibria in the five-body problem, Phys. D 127 (1999) no. 3-4, 141-145. MR1669486 (2000c:70010)

29. A. Seidenberg, Elements of the Theory of Algebraic Curves, Addison Wesley, Reading, Massachusetts (1968). MR0248139 (40:1393)

30. I. R. Shafarevich, Basic Algebraic Geometry 1, Varieties in Projective Space, Springer Verlag, Berlin-Heidelberg-New York (1994). MR,1328833 (95m:14001)

31. J. L. Synge, On the motion of three vortices, Can. J. Math. 1 (1949) 257-270. MR0030841 $(11: 61 \mathrm{c})$

32. Sir W. Thomson (Lord Kelvin), On vortex atoms, Proc. R. Soc. Edinburgh 6 (1867) 94-105.

33. J. Verschelde, 1999, Algorithm 795: PHCpack: A General-Purpose Solver for Polynomial Systems by Homotopy Continuation, ACM Trans. Math. Softw. 25, 251-276.

34. S. Wolfram, Mathematica, version 5.0.1.0, Wolfram Research, Inc.

School of Mathematics, University of Minnesota, Duluth, Minnesota 55812

E-mail address: mhampton@d.umn.edu

School of Mathematics, University of Minnesota, Minneapolis, Minnesota 55455

E-mail address: rick@math.umn.edu 